Review Article

\title{
Preclinical and Clinical Therapeutic Strategies Affecting Tumor-Associated Macrophages in Hepatocellular Carcinoma
}

\author{
H. Degroote, ${ }^{1,2}$ A. Van Dierendonck, ${ }^{1}$ A. Geerts, ${ }^{1,2}$ H. Van Vlierberghe, ${ }^{1,2}$ \\ and L. Devisscher $\mathbb{D}^{1,3}$ \\ ${ }^{1}$ Department of Gastroenterology and Hepatology, Ghent University, Belgium \\ ${ }^{2}$ Ghent University Hospital, Building K12 First Floor IE, Corneel Heymanslaan 10, 9000 Ghent, Belgium \\ ${ }^{3}$ Department Basic and Applied Medical Sciences, Ghent University, Belgium
}

Correspondence should be addressed to L. Devisscher; lindsey.devisscher@ugent.be

Received 6 July 2018; Revised 7 September 2018; Accepted 27 September 2018; Published 16 October 2018

Guest Editor: Monica Neagu

Copyright (c) 2018 H. Degroote et al. This is an open access article distributed under the Creative Commons Attribution License, which permits unrestricted use, distribution, and reproduction in any medium, provided the original work is properly cited.

\begin{abstract}
Hepatocellular carcinoma (HCC) most often develops in patients with underlying liver disease characterized by chronic nonresolving inflammation. Tumor-associated macrophages (TAMs) are one of the most abundant immune cell populations within the tumoral microenvironment. As key actors of cancer-related inflammation, they promote tumor growth by suppression of effective anticancer immunity, stimulation of angiogenesis, and tissue remodeling. Therefore, they have become an attractive and promising target for immunotherapy. The heterogeneity of TAM subtypes and their origin and dynamic phenotype during the initiation and progression of HCC has been partially unraveled and forms the base for the development of therapeutic agents. Current approaches are aimed at decreasing the population of TAMs by depleting macrophages present in the tumor, blocking the recruitment of bone marrow-derived monocytes, and/or functionally reprogramming TAMs to antitumoral behavior. In this review, the preclinical evolution and hitherto clinical trials for TAM-targeted therapy in HCC will be highlighted.
\end{abstract}

\section{Introduction}

Hepatocellular carcinoma (HCC) lesions usually arise in patients with underlying liver cirrhosis, characterized by a chronic, dysregulated inflammatory environment that predisposes to cancer initiation. In chronic liver diseases, a predominantly proinflammatory state switches to persistent systemic inflammation and immune cell stimulation but with impairment of specific immune responses such as phagocytosis and antigen-presenting ability, a condition which is called cirrhosis-associated immune dysfunction. This is an important difference with an acute inflammatory response, regarded as protective and beneficial in the acute setting of liver damage and resolution. Chronic inflammation drives indeed a maladaptive tissue repair reaction and eventually results in the development of dysplastic nodules and cancer [1-4].

The central functions of macrophages during chronic liver diseases include the perpetuation of chronic inflammation and hepatocyte injury, activation of hepatic stellate cells with subsequent fibrogenesis, and support of tumor development by providing cytokines, chemokines, growth factors, and matrix metalloproteases, all of which are factors that favor angiogenesis and tumor cell proliferation and protection from cancer cell apoptosis and metastasis $[5,6]$. Thus, hepatic macrophages provide a tumor-prone inflammatory microenvironment and at the same time respond to tumor and other stromal cell-derived signals to actively facilitate HCC progression $[1,7,8]$. Tumor-associated macrophages (TAMs) also stimulate tumor growth by acting as immune suppressor cells of the adaptive system. Not only do TAMs exhibit generally low antigen-presenting and costimulating capacity but they also actively support cancer cells to evade antitumor immunity by secreting anti-inflammatory cytokines and activating $\mathrm{T}$ cell checkpoint blockade. In this regard, tumor-infiltrating monocytes in HCC express high level of programmed cell death-ligand 1 (PD-L1) that binds with PD-1 on CD8+ T cells and suppresses antitumoral cytotoxic $\mathrm{T}$ cell responses [9-11]. This overall immune-suppressive 
effect is reinforced by cross-talk with other important immune cells in the tumoral microenvironment, such as myeloid-derived suppressor cells (MDSC) and regulatory T cells (Tregs). Besides t5he suppression of cytotoxic T cells, MDSC and Tregs contribute to the dysfunctional state of dendritic cells (DCs) [1, 12-14].

Since TAMs influence various aspects of cancer progression, novel strategies to treat HCC are aimed at targeting tumor-promoting macrophages. New therapeutic development is an urgent unmet need as options are still limited for patients with advanced HCC or earlier stage progressing upon or patients unsuitable for locoregional therapies. Nowadays, cancer immunotherapy mainly focuses on immune checkpoint inhibitors. After the observed efficacy in other solid tumors, clinical trials are currently ongoing to evaluate the utility in patients with HCC. Based on promising data in the phase I/II CheckMate-040 trial, immune therapy with nivolumab (anti-PD-1 antibody) has received FDA approval in second-line treatment [15]. However, only $20 \%$ of patients are responsive. In solid tumors, recent studies suggest that the efficacy could be enhanced using coordinated strategies to counteract the TAM-dependent impairment of immune adaptive responses [16-18].

Although the clinical application of a TAM-targeted approach still has to be determined, a number of experimental preclinical studies have shown promising effects. Most studies involve other solid tumors and are described elsewhere [19-24]. In this review, the preclinical progress and limited clinical trials affecting TAMs in HCC therapy will be highlighted. Furthermore, the encountered challenges are discussed in relation to fundamental insights into the heterogeneity of TAM subtypes and their origin and dynamic phenotype and function during the initiation and progression of HCC. Lastly, we elaborate on the potential contributive effect of combinational therapies with clinically used therapies such as sorafenib and immune checkpoint inhibitors.

\section{Definition and Origin of TAMs in HCC}

Liver macrophages consist of ontogenically distinct populations, namely, the resident Kupffer cells (KCs) and monocyte-derived macrophages (Mo-Mfs). Kupffer cells are self-renewing and nonmigratory phagocytes. They originate from yolk sac-derived specific progenitor cells that seed the liver during embryogenesis. In the tumoral microenvironment, chemokines secreted by malignant and stromal cells recruit bone marrow-derived Ly- $6 c^{\text {hi }}$ monocytes. These infiltrating monocytes subsequently give rise to large numbers of Mo-Mfs. Monocyte-derived macrophages further differentiate and can replace and acquire a phenotype that is almost indistinguishable from resident KCs under specific circumstances [25-30]. After infiltration, Mo-Mfs even seem to acquire the ability to proliferate [27]. It is however unclear if they are able to sustain the number of TAMs in tumor lesions independently from recruitment. As a result of this continuous transition, the compartment of hepatic myeloid cells consists of subtypes of macrophages in a different state of differentiation. Each state is associated with stereotypic alterations in cell surface marker expression, which can be used for identification. In many studies, CD68 is used as an indicator for tissue macrophages, but this marker is not sufficiently specific. More recently, two markers were proposed to distinguish between Mo-Mfs and KCs. Clec4F and Tim4 are expressed by KCs but absent from infiltrating Mo-Mfs. Additionally, these markers can be used to discriminate between KCs and recently differentiated Mo-KCs as the latter do not express Tim4 in the first week postdifferentiation. However, with time, Mo-KCs will also gain expression of Tim4 [27, 28].

It is not clear to what extent TAMs are derived from tissue-resident liver cells or only represent infiltrating bonemarrow derived Mo-Mfs. In most reports, macrophages present in the tumoral microenvironment are considered and classified as "tumor-associated macrophages." Although KCs were initially thought to be only involved in antitumor immunity, there is substantial evidence that suggests that KCs are part of the TAM population and enhance tumor progression [3, 31-33]. KCs are triggered by damage-associated molecular patterns (DAMPs) released from damaged liver cells and pathogen-associated molecular patterns (PAMPs), mostly derived from the gut due to alterations in gut microbiota composition and/or increased intestinal permeability. The liver is supplied with blood via the portal vein from the intestinal tract and via hepatic arteries from the blood circulation. As such, KCs in the liver sinusoids are exposed to bacteria and associated toxins from the bloodstream [34]. DAMPs and PAMPs interact with pattern recognition receptors (PRR) on $\mathrm{KC}$ or directly on activating inflammasomes [34]. For example, the interaction of lipopolysaccharide (LPS) with Toll-like receptor 4 (TLR-4) on KCs showed stimulation of cancer-promoting signaling pathways in mice [35].

\section{Phenotype and Function of TAMs in HCC}

Defining TAMs as one population has limitations as shown in the contradictory results of prognostic studies, summarized in Table 1. This is mostly due to an overgeneralized definition of TAMs and indicates the need for further subdivision according to their polarization. Polarization refers to how macrophages have been activated as they can rapidly adapt to their phenotype according to signals derived from the hepatic microenvironment. Macrophages have been assigned a classically activated (proinflammatory) M1 state triggered by interferon $-\gamma$ and/or lipopolysaccharide or an alternatively activated (anti-inflammatory) M2 state induced by IL-4. This traditional nomenclature however is derived from in vitro studies and does not represent chronic inflammation or the complex tumoral microenvironment. Moreover, the expression and secretory profile of macrophage subsets are not dichotomous and can differ according to the model and method of inducing polarization. There is also considerable difference between mouse and human cells in terms of molecules associated with macrophage polarization [28, 36-39].

The pro- and anti-inflammatory paradigm leads to the confusing assumption that in an inflammation-related tumor, an M2 phenotype would be beneficial. However, during tumor progression in HCC, macrophage function is 
TABle 1: Prognosis of HCC according to TAM identification and polarization. Immunohistochemical staining for CD68, CD86 (M1), or CD163 and CD206 (M2) is frequently used to quantify and classify TAMs [38, 41]. Expression of the used tissue markers was determined by immunohistochemical staining. Serum sCD163 levels were measured by ELISA. Defining TAMs as solely CD68+ cells gives contradictory prognostic results. When however TAMs are subdivided for location (intra- or peritumoral) and polarization (M1- or M2-like cells), a more distinct prognostic value can be attributed. Moreover, it becomes clear from the presented studies that the presence of M2-like oriented TAMs results in a poor prognostic outcome and intratumoral M1-like TAMs correlate with good prognosis.

\begin{tabular}{|c|c|c|c|c|c|}
\hline Author & Number of patient samples & Type of sample & Marker & Definition & Prognosis \\
\hline Li et al. [42] & 101 & Intratumoral & CD68+ & TAM & Poor \\
\hline Ding et al. [43] & 137 & Intratumoral & CD68+ & TAM & Poor \\
\hline Kuang et al. [11] & 262 & Peritumoral & CD68+ & TAM & Poor \\
\hline Zhang et al. [44] & 149 & Peritumoral & CD68+ & TAM & Poor \\
\hline Zhou et al. [45] & 213 & Intratumoral & CD68+ & TAM & Poor \\
\hline Wu et al. [33] & 71 & Intratumoral & CD68+ & TAM & Poor \\
\hline Minami et al. [46] & 105 & Intratumoral & CD68+ & TAM & Poor \\
\hline Liao et al. [47] & 387 & Intratumoral & CD68+ & TAM & Not related \\
\hline Dong et al. [48] & 253 & Intratumoral & CD68+ & TAM & Not related \\
\hline Yeung et al. [49] & 93 & Intratumoral & CD68+ & TAM & Good \\
\hline Yeung et al. [49] & 93 & Peritumoral & CD68+ & TAM & Poor \\
\hline Li et al. [50] & 302 & Intratumoral & CD68+ & TAM & Good \\
\hline Liao et al. [47] & 387 & Intratumoral & CD16+ & M2-like & Poor \\
\hline Waidmann et al. [51] & 267 & Serum & sCD163+ & M2-like & Poor \\
\hline Minami et al. [46] & 105 & Intratumoral & CD163+ & M2-like & Poor \\
\hline Yeung et al. [49] & 93 & Peritumoral & CD163+ & M2-like & Poor \\
\hline Dong et al. [48] & 253 & Intratumoral & CD206+ & M2-like & Poor \\
\hline Dong et al. [48] & 253 & Intratumoral & CD86+ & M1-like & Good \\
\hline
\end{tabular}

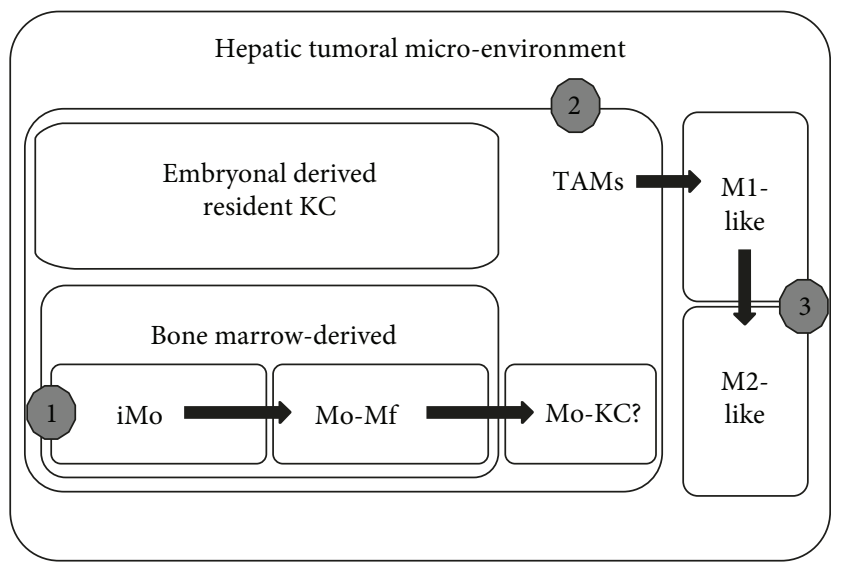

(i) Inhibiting recruitment infiltrating monocytes

(ii) Depletion of TAMs present in tumor

(iii) Reprogramming polarization of TAMs

Figure 1: Origin of TAMs in the hepatic tumoral microenvironment and related TAM-targeted strategies.

skewed from M1 to M2 phenotype [10, 40]. An antiinflammatory phenotype does not result in the resolution of inflammation but refers to the immune-deficient and immune-suppressive state of these macrophages and consequently immune evasion of cancer cells. On the other hand, a proinflammatory phenotype does not refer to inflammatory damage in an acute setting but represents a coordinated immune attack of tumor cells. Thus, the M1/M2 model is too simplistic to describe the polarization of liver macrophages in cancer. Currently, TAMs are most often defined as M1-like (leading to antitumor responses and cytotoxicity) or M2-like (tumor promotion and suppression of effective adaptive immunity) cells, taking into account the relative proportion between both characteristics as they often simultaneously express markers of both ends of the continuum [36]. The polarization of macrophages not only depends on the disease stage but also differs between tumoral nodules or within different areas of the same tumor. In human HCC, for example, most of the macrophages that are localized perivascularly are more M1like compared to the M2-like TAM in hypoxic areas $[25,38]$.

\section{TAM-Targeted Therapy in HCC}

Current approaches for TAM-targeted therapy are aimed at decreasing the population of TAMs by eliminating TAMs present in the tumor, blocking recruitment of bone marrow-derived monocytes, and/or reprogramming TAM polarization to antitumoral behavior (Figure 1).

4.1. Preclinical Studies. In the following section, only preclinical studies in mouse models using agents with a direct effect on TAMs in HCC will be discussed and are summarized in Table 2. Gene therapy or knockout models are beyond the scope of this review.

4.1.1. Depletion of TAMs. Liposomes are artificially prepared vesicles that undergo phagocytosis by macrophages after 


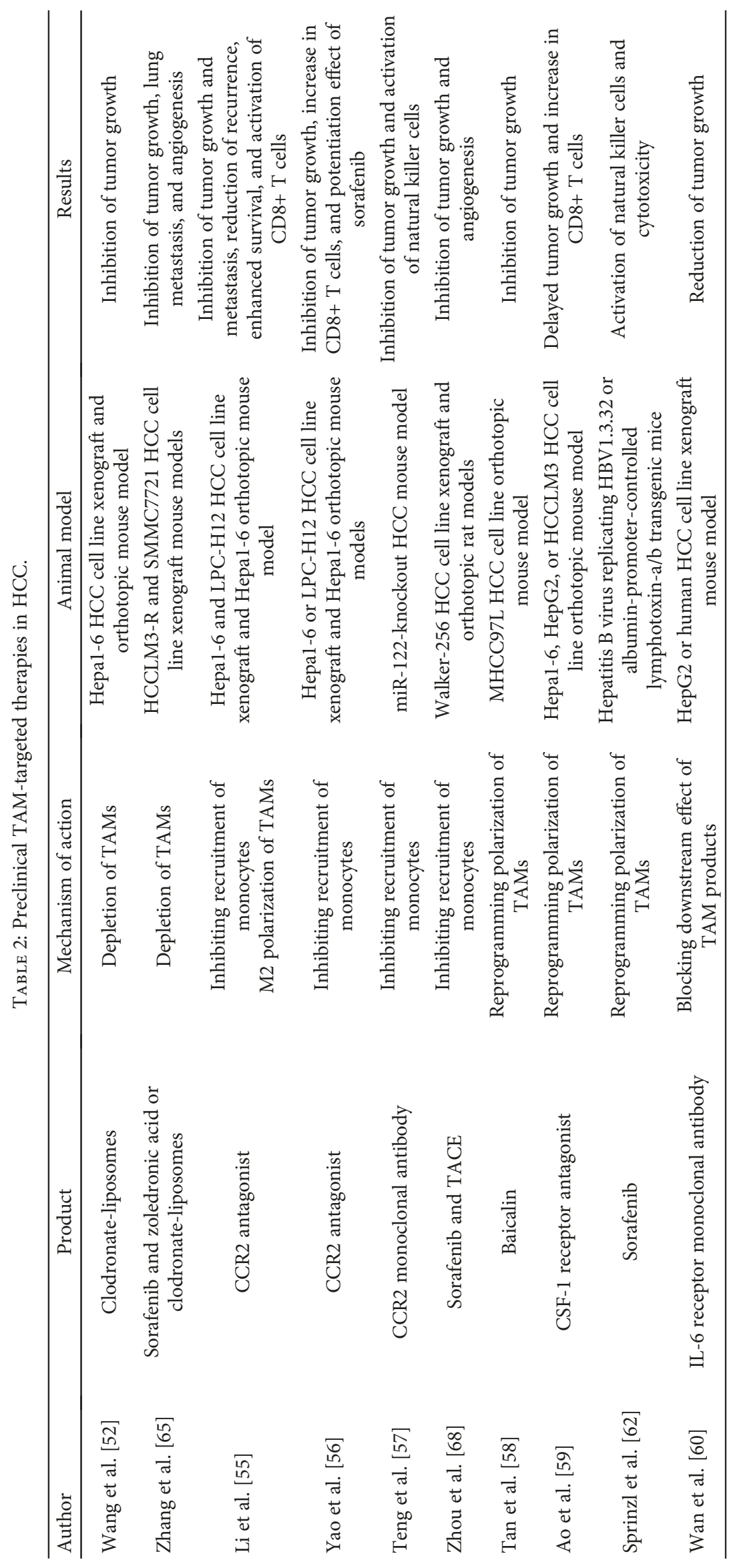


injection. They can be loaded with clodronate (a bisphosphonate used for osteoporosis) which induces apoptosis of macrophages after intracellular release from the liposomes. Administration of clodronate- (Cl2MDP) encapsulated liposomes partially depleted TAMs (defined as F4/80- and CD68-positive cells on immunohistochemical staining), resulting in reduced tumor growth in a murine Hepal-6 cell-transplanted tumor model. Not only was the total amount of TAMs reduced but also the number of M2-like TAMs in tumors of liposome-treated mice was significantly lower than that in tumors of untreated mice. In contrast, the number of M1 TAMs was not significantly affected. According to the authors, these results suggest that after depleting the majority of TAMs, the remaining macrophages might undergo a phenotypical transition [52].

Selective depletion of only tumor-promoting macrophages, not just all cells with phagocyting capacity, is an encountered difficulty in TAM-targeted therapy. An excessive reduction of nontumoral macrophages might lead to safety concerns when concomitant infections occur. The use of macrophage subset-specific markers might provide a solution and has successfully been used in the field of imaging where $\mathrm{KC}$-specific [53] or TAM-specific targeting by nanobodies coupled to SPECT or PET tracers allowed KC-specific and M2-like TAM-specific imaging, respectively [54]. Further research is warranted to see if this approach could be translated to pharmaceutical development. It must be emphasized that TAMs are strongly connected with other immune and stromal cells in the microenvironment and it is not clear to what extent other cells will compensate for their function after depletion.

4.1.2. Inhibiting Recruitment of Monocytes. The chemokine $\mathrm{C}-\mathrm{C}$ motif ligand 2 (CCL2, also referred to as monocyte chemoattractant protein 1 or $\mathrm{MCP}-1$ ) and the corresponding CCL2-CCR2 signaling axis are important targets to inhibit the recruitment of monocytes. Treatment with a CCR2 antagonist inhibited HCC tumor growth in different murine models. The therapy reduced the infiltration of blood Ly6C ${ }^{\text {high }}$ inflammatory monocytes, subsequently lowered the number of TAMs (CD11b- and F4/80-positive cells) in the HCC lesions, and reduced most of the cytokines or chemokines produced by M2-like TAMs (CD206-positive cells). Moreover, the reduced number of remaining TAM shifted towards M1 phenotype. The CCR2 antagonist also supported tumor-infiltrated CD8+ T cells by blocking TAM-mediated immunosuppression $[55,56]$. In addition, Teng et al. showed the tumor-inhibiting effect of a CCL2 neutralizing antibody by reducing the population of inflammatory myeloid cells in a HCC mouse model [57]. Although several chemokines are involved in attracting monocytes and targeting one pathway might not completely eliminate recruitment, blocking the CCL2-CCR2 seems to be effective in the inhibition of HCC growth.

Infiltration of monocytes is considered the most important source of TAMs in the tumoral microenvironment. It is still unclear if TAMs are able to sustain their number (or least partially) in tumors by proliferation independently from recruitment or how long TAMs survive in the tumoral microenvironment. Related to this issue, effective timing to start inhibiting recruitment of monocytes can be debated as during early stages, TAMs can also exert an antitumoral function.

4.1.3. Reprogramming Polarization of TAMs. Oral administration of baicalin, a natural flavonoid present in several medicinal plants, inhibited growth of HCC lesions in an orthotopic mouse model by initiating TAM reprogramming to an M1-like phenotype with proinflammatory cytokine production. Coculturing of HCC cells with baicalin-treated macrophages resulted in reduced proliferation and motility in vitro [58].

Colony-stimulating factor-1 (CSF-1) and its receptor, CSF-1R, regulate the differentiation and function of macrophages. CSF-1R blockade by a competitive inhibitor significantly delayed tumor growth in murine xenograft models. The compound inhibited the proliferation of macrophages in vitro, but macrophage infiltration was not decreased in vivo. Thus, the effect is not mediated by TAM depletion. Gene expression profiling showed that TAMs in the treated tumors are polarized towards an M1-like phenotype [59].

An imbalance towards M1-like macrophages might theoretically be harmful by inducing toxicity and inflammatory conditions. In the mentioned studies, no toxic effects were observed but further studies are necessary.

4.1.4. Blocking the Downstream Effect of TAM Products. TAMs represent a major paracrine IL-6 source during HCC progression, and autocrine IL- 6 contributed significantly to HCC initiation from HCC progenitor cells. Blockade of IL-6 signaling using tocilizumab, an anti-IL-6 receptor antibody approved by the FDA for the treatment of rheumatoid arthritis, was able to inhibit TAM-stimulated activity of cancer stem cells in vitro and in vivo [60].

4.2. Preclinical Therapy Affecting TAMs with Currently Used Clinical Therapies. Sorafenib, an antiangiogenic oral multikinase inhibitor, is currently the standard first-line systemic treatment approved by the US Food and Drug Administration (FDA) and European Medicines Agency (EMA) for patients with advanced HCC. Preclinical studies show that sorafenib interferes with the polarization of TAMs and their cytokine production. In a HepG2 HCC cell line, sorafenib inhibited polarized macrophage-induced epithelial-mesenchymal transition and migration of HCC cells [61]. Administration of sorafenib reduced M2-like TAMs, inhibited their immunesuppressive effect, and stimulated antitumor natural killer (NK) cell responses in both HCC models [62]. In addition, Sprinzl et al. [63] demonstrated a decrease in CD163 serum concentration in 21 patients with HCC during treatment with sorafenib. This finding suggests that Sorafenib suppressed M2 activation in HCC patients, since soluble sCD163 is shedded into serum by activated macrophages and can serve as an indicator to follow M2 macrophage responses [51, 64]. Together, these findings indicate that macrophage modulation contributes to the anticancer activity of sorafenib.

Interestingly, the combination of sorafenib with TAM-targeting agents such as clodronate-loaded liposomes 
and zoledronic acid (another bisphosphonate used for the treatment of bone metastasis) augmented the inhibitory effect of sorafenib on tumor angiogenesis, growth, and metastasis in HCC xenograft mouse models [65]. A phase II study of sorafenib combined with zoledronic acid in advanced HCC has been conducted (NCT01259193), but no results have been published yet. Besides depletion of macrophages, nitrogen-containing bisphosphonates (such as zoledronic acid) activate $\gamma \delta \mathrm{T}$ cells, potentiating their antitumor function. This immunomodulatory effect of zoledronic acid on $\gamma \delta \mathrm{T}$ cells is exerted through direct or indirect interaction induced by TAMs that endocytose bisphosphonate-encapsulated liposomes [66, 67]. Not only is this effect shown for sorafenib but also the combination of locoregional therapy such as transarterial chemoembolization (TACE) and zoledronic acid treatment showed enhanced therapeutic efficacy with inhibition of TAM infiltration (F4/80+) and tumor angiogenesis in a rat HCC model [68]. The enhanced efficacy of sorafenib together with a CCR2 antagonist to inhibit monocyte infiltration has been shown in a murine HCC model [56].

The effect of checkpoint blockade immunotherapy on TAMs has been shown for other solid tumors, but no data are available in HCC. Blockade of PD-1 in vivo reduced tumor growth and extended the survival in a colorectal cancer mouse model by polarization of TAMs to a phagocytic phenotype [69]. The efficacy of anticytotoxic T lymphocyte-associated antigen 4 (CTLA-4) monoclonal antibodies in a melanoma mouse model and humans is codefined by elimination of regulatory $\mathrm{T}$ cells by TAM targeting via antibody-dependent cellular cytotoxicity [16-18, 45]. Also, in a pancreatic cancer mouse model, the combination of depleting M2-like TAMs and repolarization towards antitumoral behavior through blockage of CSF-1/CSF-1R and immunotherapy (PD-1 and CTLA-4 antagonists) reduced tumor progression [18].

4.3. Clinical Trials. Glypican-3 is a proteoglycan that is attached to the cell surface and plays an important role in cellular growth, differentiation, and migration. Glypican-3 is highly expressed in HCC tissue and correlates with poor prognosis. It is considered a tumor-derived carcinoembryonic antigen. For example, expression of glypican-3 was associated with upregulation of CCL5, CCL3, and CSF-1 in a HCC xenograft model [70], all of which are chemokines that have been shown to enhance the recruitment of TAMs. Glypican-3 antibodies have been tested in small phase I trials for advanced HCC with promising results (in 13 and 20 patients, respectively). The antibody is well tolerated, and preliminary antitumor activity shows a threefold prolongation of the median time to progression in treated patients with advanced HCC (Child-Pugh A or B cirrhosis) [71, 72]. No phase II trials are currently registered with glypican-3 antibodies for HCC.

\section{Conclusion}

The tumor-promoting cascade of initial injury recognition, amplification of inflammation by monocyte recruitment, and context-dependent differentiation into functionally distinct macrophage populations in the liver offers different approaches for therapeutic interventions in HCC. Although the clinical application of TAM-targeted therapy is still in its infancy, a number of preclinical studies in HCC murine models have shown promising results. The most important obstacles to overcome are firstly the specificity of depleting only protumoral TAMs while not affecting (or even enhancing) antitumor immunity and secondly the perfect balance of their polarization towards antitumoral behavior without toxicity and side effects. The observed potential contributive effect of immune checkpoint inhibitors on solid tumors and currently used clinical therapies for HCC such as sorafenib is encouraging and must be further explored.

\section{Abbreviations}

CSF-1: Colony-stimulating factor-1

CSF-1R: Colony-stimulating factor-1 receptor

CTLA-4: Cytotoxic T lymphocyte-associated antigen 4

DAMP: Damage-associated molecular patterns

HCC: Hepatocellular carcinoma

KCs: $\quad$ Kupffer cells

Mo-Mfs: Monocyte-derived macrophages

Mo-KCs: Monocyte-derived Kupffer cells

PAMPs: Pathogen-associated molecular patterns

PD-1: $\quad$ Programmed cell death 1

PD-L1: Programmed cell death-ligand 1

TAMs: Tumor-associated macrophages.

\section{Conflicts of Interest}

The authors declare no conflict of interest.

\section{Acknowledgments}

$\mathrm{HD}$ and LD are sponsored by a fund from Kom op tegen Kanker (Stand up to Cancer), a Flemish cancer society. LD received a postdoctoral fellowship from the Special Research Funds (BOF) from Ghent University. AV is sponsored by the Special Research Funds (BOF) from Ghent University. HVV and AG are sponsored by the Research Foundation Flanders (FWO).

\section{References}

[1] V. Hernandez-Gea, S. Toffanin, S. L. Friedman, and J. M. Llovet, "Role of the microenvironment in the pathogenesis and treatment of hepatocellular carcinoma," Gastroenterology, vol. 144, no. 3, pp. 512-527, 2013.

[2] R. Ostuni, F. Kratochvill, P. J. Murray, and G. Natoli, "Macrophages and cancer: from mechanisms to therapeutic implications," Trends in Immunology, vol. 36, no. 4, pp. 229-239, 2015.

[3] R. N. Aravalli, "Role of innate immunity in the development of hepatocellular carcinoma," World Journal of Gastroenterology, vol. 19, no. 43, pp. 7500-7514, 2013.

[4] A. Albillos, M. Lario, and M. Alvarez-Mon, "Cirrhosisassociated immune dysfunction: distinctive features and 
clinical relevance," Journal of Hepatology, vol. 61, no. 6, pp. 1385-1396, 2014.

[5] D. Capece, M. Fischietti, D. Verzella et al., "The inflammatory microenvironment in hepatocellular carcinoma: a pivotal role for tumor-associated macrophages," BioMed Research International, vol. 2013, Article ID 187204, 15 pages, 2013.

[6] K. Shirabe, Y. Mano, J. Muto et al., "Role of tumor-associated macrophages in the progression of hepatocellular carcinoma," Surgery Today, vol. 42, no. 1, pp. 1-7, 2012.

[7] S. D. Wu, Y. S. Ma, Y. Fang, L. L. Liu, D. Fu, and X. Z. Shen, "Role of the microenvironment in hepatocellular carcinoma development and progression," Cancer Treatment Reviews, vol. 38, no. 3, pp. 218-225, 2012.

[8] F. Heindryckx and P. Gerwins, "Targeting the tumor stroma in hepatocellular carcinoma," World Journal of Hepatology, vol. 7, no. 2, pp. 165-176, 2015.

[9] A. J. Petty and Y. Yang, "Tumor-associated macrophages: implications in cancer immunotherapy," Immunotherapy, vol. 9, no. 3, pp. 289-302, 2017.

[10] J. Prieto, I. Melero, and B. Sangro, "Immunological landscape and immunotherapy of hepatocellular carcinoma," Nature Reviews Gastroenterology \& Hepatology, vol. 12, no. 12, pp. 681-700, 2015.

[11] D. M. Kuang, Q. Zhao, C. Peng et al., “Activated monocytes in peritumoral stroma of hepatocellular carcinoma foster immune privilege and disease progression through PD-L1," Journal of Experimental Medicine, vol. 206, no. 6, pp. 13271337, 2009.

[12] N. Schmidt, C. Neumann-Haefelin, and R. Thimme, "Cellular immune responses to hepatocellular carcinoma: lessons for immunotherapy," Digestive Diseases, vol. 30, no. 5, pp. 483491, 2012.

[13] P. Patel, S. E. Schutzer, and N. Pyrsopoulos, "Immunobiology of hepatocarcinogenesis: ways to go or almost there?," World Journal of Gastrointestinal Pathophysiology, vol. 7, no. 3, pp. 242-255, 2016.

[14] N. Nishida and M. Kudo, "Immunological microenvironment of hepatocellular carcinoma and its clinical implication," Oncology, vol. 92, no. 1, pp. 40-49, 2017.

[15] European Association for the Study of the Liver, "EASL clinical practice guidelines: management of hepatocellular carcinoma," Journal of Hepatology, vol. 69, no. 1, pp. 182-236, 2018.

[16] T. R. Simpson, F. Li, W. Montalvo-Ortiz et al., "Fc-dependent depletion of tumor-infiltrating regulatory $\mathrm{T}$ cells co-defines the efficacy of anti-CTLA-4 therapy against melanoma," Journal of Experimental Medicine, vol. 210, no. 9, pp. 1695-1710, 2013.

[17] E. Romano, M. Kusio-Kobialka, P. G. Foukas et al., "Ipilimumab-dependent cell-mediated cytotoxicity of regulatory $\mathrm{T}$ cells ex vivo by nonclassical monocytes in melanoma patients," Proceedings of the National Academy of Sciences of the United States of America, vol. 112, no. 19, pp. 6140-6145, 2015.

[18] Y. Zhu, B. L. Knolhoff, M. A. Meyer et al., "CSF1/CSF1R blockade reprograms tumor-infiltrating macrophages and improves response to $\mathrm{T}$-cell checkpoint immunotherapy in pancreatic cancer models," Cancer Research, vol. 74, no. 18, pp. 50575069, 2014.

[19] A. Mantovani, F. Marchesi, A. Malesci, L. Laghi, and P. Allavena, "Tumour-associated macrophages as treatment targets in oncology," Nature Reviews Clinical Oncology, vol. 14, no. 7, pp. 399-416, 2017.

[20] Q. Guo, Z. Jin, Y. Yuan et al., "New mechanisms of tumorassociated macrophages on promoting tumor progression: recent research advances and potential targets for tumor immunotherapy," Journal of Immunology Research, vol. 2016, Article ID 9720912, 12 pages, 2016.

[21] S. Bonelli, X. Geeraerts, E. Bolli et al., "Beyond the M-CSF receptor - novel therapeutic targets in tumor-associated macrophages," The FEBS Journal, vol. 285, no. 4, pp. 777-787, 2018.

[22] A. Weigert, D. Sekar, and B. Brune, "Tumor-associated macrophages as targets for tumor immunotherapy," Immunotherapy, vol. 1, no. 1, pp. 83-95, 2009.

[23] K. Sawa-Wejksza and M. Kandefer-Szerszen, "Tumorassociated macrophages as target for antitumor therapy," Archivum Immunologiae et Therapiae Experimentalis, vol. 66, no. 2, pp. 97-111, 2018.

[24] M. Majety, V. Runza, C. Lehmann, S. Hoves, and C. H. Ries, “A drug development perspective on targeting tumor-associated myeloid cells," The FEBS Journal, vol. 285, no. 4, pp. 763776, 2018.

[25] K. Movahedi and J. A. Van Ginderachter, "The ontogeny and microenvironmental regulation of tumor-associated macrophages," Antioxidants \& Redox Signaling, vol. 25, no. 14, pp. 775-791, 2016.

[26] F. Tacke, T. Luedde, and C. Trautwein, "Inflammatory pathways in liver homeostasis and liver injury," Clinical Reviews in Allergy and Immunology, vol. 36, no. 1, pp. 4-12, 2009.

[27] C. L. Scott, F. Zheng, P. de Baetselier et al., "Bone marrowderived monocytes give rise to self-renewing and fully differentiated Kupffer cells," Nature Communications, vol. 7, article 10321, 2016.

[28] L. Beattie, A. Sawtell, J. Mann et al., "Bone marrow-derived and resident liver macrophages display unique transcriptomic signatures but similar biological functions," Journal of Hepatology, vol. 65, no. 4, pp. 758-768, 2016.

[29] A. Sica, P. Invernizzi, and A. Mantovani, "Macrophage plasticity and polarization in liver homeostasis and pathology," Hepatology, vol. 59, no. 5, pp. 2034-2042, 2014.

[30] L. Devisscher, C. L. Scott, S. Lefere et al., "Non-alcoholic steatohepatitis induces transient changes within the liver macrophage pool," Cellular Immunology, vol. 322, pp. 74-83, 2017.

[31] J. D. Yang, I. Nakamura, and L. R. Roberts, "The tumor microenvironment in hepatocellular carcinoma: current status and therapeutic targets," Seminars in Cancer Biology, vol. 21, no. 1, pp. 35-43, 2011.

[32] J. Wu, J. Li, R. Salcedo, N. F. Mivechi, G. Trinchieri, and A. Horuzsko, "The proinflammatory myeloid cell receptor TREM-1 controls Kupffer cell activation and development of hepatocellular carcinoma," Cancer Research, vol. 72, no. 16, pp. 3977-3986, 2012.

[33] K. Wu, I. Kryczek, L. Chen, W. Zou, and T. H. Welling, "Kupffer cell suppression of $\mathrm{CD}^{+} \mathrm{T}$ cells in human hepatocellular carcinoma is mediated by $\mathrm{B} 7-\mathrm{H} 1 /$ programmed death- 1 interactions," Cancer Research, vol. 69, no. 20, pp. 8067-8075, 2009.

[34] L. X. Yu and R. F. Schwabe, "The gut microbiome and liver cancer: mechanisms and clinical translation," Nature Reviews Gastroenterology \& Hepatology, vol. 14, no. 9, pp. 527-539, 2017. 
[35] D. H. Dapito, A. Mencin, G. Y. Gwak et al., "Promotion of hepatocellular carcinoma by the intestinal microbiota and TLR4," Cancer Cell, vol. 21, no. 4, pp. 504-516, 2012.

[36] P. J. Murray, "Macrophage polarization," Annual Review of Physiology, vol. 79, no. 1, pp. 541-566, 2017.

[37] F. Tacke, "Targeting hepatic macrophages to treat liver diseases," Journal of Hepatology, vol. 66, no. 6, pp. 1300-1312, 2017.

[38] D. Laoui, E. van Overmeire, K. Movahedi et al., "Mononuclear phagocyte heterogeneity in cancer: different subsets and activation states reaching out at the tumor site," Immunobiology, vol. 216, no. 11, pp. 1192-1202, 2011.

[39] E. Van Overmeire, D. Laoui, J. Keirsse, J. A. Van Ginderachter, and A. Sarukhan, "Mechanisms driving macrophage diversity and specialization in distinct tumor microenvironments and parallelisms with other tissues," Frontiers in Immunology, vol. 5, p. 127, 2014.

[40] R. D. Schreiber, L. J. Old, and M. J. Smyth, "Cancer immunoediting: integrating immunity's roles in cancer suppression and promotion," Science, vol. 331, no. 6024, pp. 1565-1570, 2011.

[41] L. A. Elliott, G. A. Doherty, K. Sheahan, and E. J. Ryan, "Human tumor-infiltrating myeloid cells: phenotypic and functional diversity," Frontiers in Immunology, vol. 8, p. 86, 2017.

[42] L. Li, J. Yan, J. Xu et al., "CXCL17 expression predicts poor prognosis and correlates with adverse immune infiltration in hepatocellular carcinoma," PLoS One, vol. 9, no. 10, article e110064, 2014.

[43] T. Ding, J. Xu, F. Wang et al., "High tumor-infiltrating macrophage density predicts poor prognosis in patients with primary hepatocellular carcinoma after resection," Human Pathology, vol. 40, no. 3, pp. 381-389, 2009.

[44] Q. B. Zhang, Q. A. Jia, H. Wang et al., "High-mobility group protein box1 expression correlates with peritumoral macrophage infiltration and unfavorable prognosis in patients with hepatocellular carcinoma and cirrhosis," BMC Cancer, vol. 16, no. 1, p. 880, 2016.

[45] J. Zhou, T. Ding, W. Pan, L. Y. Zhu, L. Li, and L. Zheng, "Increased intratumoral regulatory $\mathrm{T}$ cells are related to intratumoral macrophages and poor prognosis in hepatocellular carcinoma patients," International Journal of Cancer, vol. 125, no. 7, pp. 1640-1648, 2009.

[46] K. Minami, K. Hiwatashi, S. Ueno et al., "Prognostic significance of CD68, CD163 and folate receptor- $\beta$ positive macrophages in hepatocellular carcinoma," Experimental and Therapeutic Medicine, vol. 15, no. 5, pp. 4465-4476, 2018.

[47] R. Liao, N. Jiang, Z. W. Tang et al., "Systemic and intratumoral balances between monocytes/macrophages and lymphocytes predict prognosis in hepatocellular carcinoma patients after surgery," Oncotarget, vol. 7, no. 21, pp. 30951-30961, 2016.

[48] P. Dong, L. Ma, L. Liu et al., "CD $86^{+} / \mathrm{CD} 206^{+}$, diametrically polarized tumor-associated macrophages, predict hepatocellular carcinoma patient prognosis," International Journal of Molecular Sciences, vol. 17, no. 3, p. 320, 2016.

[49] O. W. H. Yeung, C. M. Lo, C. C. Ling et al., "Alternatively activated (M2) macrophages promote tumour growth and invasiveness in hepatocellular carcinoma," Journal of Hepatology, vol. 62, no. 3, pp. 607-616, 2015.

[50] Y. W. Li, S. J. Qiu, J. Fan et al., “Tumor-infiltrating macrophages can predict favorable prognosis in hepatocellular carcinoma after resection," Journal of Cancer Research and Clinical Oncology, vol. 135, no. 3, pp. 439-449, 2009.

[51] O. Waidmann, V. Köberle, D. Bettinger et al., "Diagnostic and prognostic significance of cell death and macrophage activation markers in patients with hepatocellular carcinoma," Journal of Hepatology, vol. 59, no. 4, pp. 769-779, 2013.

[52] B. Wang, Q. Li, L. Qin, S. Zhao, J. Wang, and X. Chen, “Transition of tumor-associated macrophages from MHC class $\mathrm{II}^{\mathrm{h}}$ to MHC class II $^{\text {low }}$ mediates tumor progression in mice," BMC Immunology, vol. 12, no. 1, p. 43, 2011.

[53] F. Zheng, A. Sparkes, P. de Baetselier et al., "Molecular imaging with Kupffer cell-targeting nanobodies for diagnosis and prognosis in mouse models of liver pathogenesis," Molecular Imaging and Biology, vol. 19, no. 1, pp. 49-58, 2017.

[54] K. Movahedi, S. Schoonooghe, D. Laoui et al., "Nanobodybased targeting of the macrophage mannose receptor for effective in vivo imaging of tumor-associated macrophages," Cancer Research, vol. 72, no. 16, pp. 4165-4177, 2012.

[55] X. Li, W. Yao, Y. Yuan et al., "Targeting of tumour-infiltrating macrophages via CCL2/CCR2 signalling as a therapeutic strategy against hepatocellular carcinoma," Gut, vol. 66, no. 1, pp. 157-167, 2017.

[56] W. Yao, Q. Ba, X. Li et al., “A natural CCR2 antagonist relieves tumor-associated macrophage-mediated immunosuppression to produce a therapeutic effect for liver cancer," eBioMedicine, vol. 22, pp. 58-67, 2017.

[57] K.-Y. Teng, J. Han, X. Zhang et al., "Blocking the Ccl2-Ccr2 axis using Ccl2 neutralizing antibody is an effective therapy for hepatocellular cancer in a mouse model," Molecular Cancer Therapeutics, vol. 16, no. 2, pp. 312-322, 2017.

[58] H. Y. Tan, N. Wang, K. Man, S. W. Tsao, C. M. Che, and Y. Feng, "Autophagy-induced RelB/p52 activation mediates tumour-associated macrophage repolarisation and suppression of hepatocellular carcinoma by natural compound baicalin," Cell Death \& Disease, vol. 6, no. 10, p. e1942, 2015.

[59] J. Y. Ao, X. D. Zhu, Z. T. Chai et al., "Colony-stimulating factor 1 receptor blockade inhibits tumor growth by altering the polarization of tumor-associated macrophages in hepatocellular carcinoma," Molecular Cancer Therapeutics, vol. 16, no. 8, pp. 1544-1554, 2017.

[60] S. Wan, E. Zhao, I. Kryczek et al., "Tumor-associated macrophages produce interleukin 6 and signal via STAT3 to promote expansion of human hepatocellular carcinoma stem cells," Gastroenterology, vol. 147, no. 6, pp. 1393-1404, 2014.

[61] Y. R. Deng, W. B. Liu, Z. X. Lian, X. Li, and X. Hou, "Sorafenib inhibits macrophage-mediated epithelial-mesenchymal transition in hepatocellular carcinoma," Oncotarget, vol. 7, no. 25, pp. 38292-38305, 2016.

[62] M. F. Sprinzl, F. Reisinger, A. Puschnik et al., "Sorafenib perpetuates cellular anticancer effector functions by modulating the crosstalk between macrophages and natural killer cells," Hepatology, vol. 57, no. 6, pp. 2358-2368, 2013.

[63] M. F. Sprinzl, A. Puschnik, A. M. Schlitter et al., "Sorafenib inhibits macrophage-induced growth of hepatoma cells by interference with insulin-like growth factor-1 secretion," Journal of Hepatology, vol. 62, no. 4, pp. 863-870, 2015.

[64] H. J. Møller, "Soluble CD163," Scandinavian Journal of Clinical and Laboratory Investigation, vol. 72, no. 1, pp. 1-13, 2012.

[65] W. Zhang, X. D. Zhu, H. C. Sun et al., "Depletion of tumorassociated macrophages enhances the effect of sorafenib in 
metastatic liver cancer models by antimetastatic and antiangiogenic effects," Clinical Cancer Research, vol. 16, no. 13, pp. 3420-3430, 2010.

[66] S. Galluzzo, D. Santini, B. Vincenzi et al., "Immunomodulating role of bisphosphonates on human gamma delta T cells: an intriguing and promising aspect of their antitumour activity," Expert Opinion on Therapeutic Targets, vol. 11, no. 7, pp. 941-954, 2007.

[67] H. H. Van Acker, S. Anguille, Y. Willemen, E. L. Smits, and V. F. Van Tendeloo, "Bisphosphonates for cancer treatment: mechanisms of action and lessons from clinical trials," Pharmacology \& Therapeutics, vol. 158, pp. 24-40, 2016.

[68] D. Y. Zhou, J. Qin, J. Huang et al., "Zoledronic acid inhibits infiltration of tumor-associated macrophages and angiogenesis following transcatheter arterial chemoembolization in rat hepatocellular carcinoma models," Oncology Letters, vol. 14, no. 4, pp. 4078-4084, 2017.

[69] S. R. Gordon, R. L. Maute, B. W. Dulken et al., "PD-1 expression by tumour-associated macrophages inhibits phagocytosis and tumour immunity," Nature, vol. 545, no. 7655, pp. 495499, 2017.

[70] H. Takai, M. Ashihara, T. Ishiguro et al., "Involvement of glypican-3 in the recruitment of M2-polarized tumorassociated macrophages in hepatocellular carcinoma," Cancer Biology \& Therapy, vol. 8, no. 24, pp. 2329-2338, 2009.

[71] M. Ikeda, S. Ohkawa, T. Okusaka et al., "Japanese phase I study of GC33, a humanized antibody against glypican-3 for advanced hepatocellular carcinoma," Cancer Science, vol. 105, no. 4, pp. 455-462, 2014.

[72] A. X. Zhu, P. J. Gold, A. B. el-Khoueiry et al., "First-in-man phase I study of GC33, a novel recombinant humanized antibody against glypican-3, in patients with advanced hepatocellular carcinoma," Clinical Cancer Research, vol. 19, no. 4, pp. 920-928, 2013. 


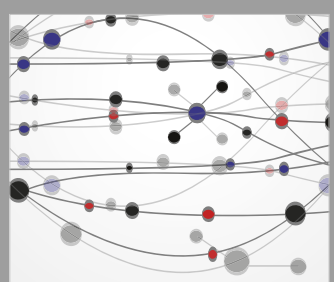

The Scientific World Journal
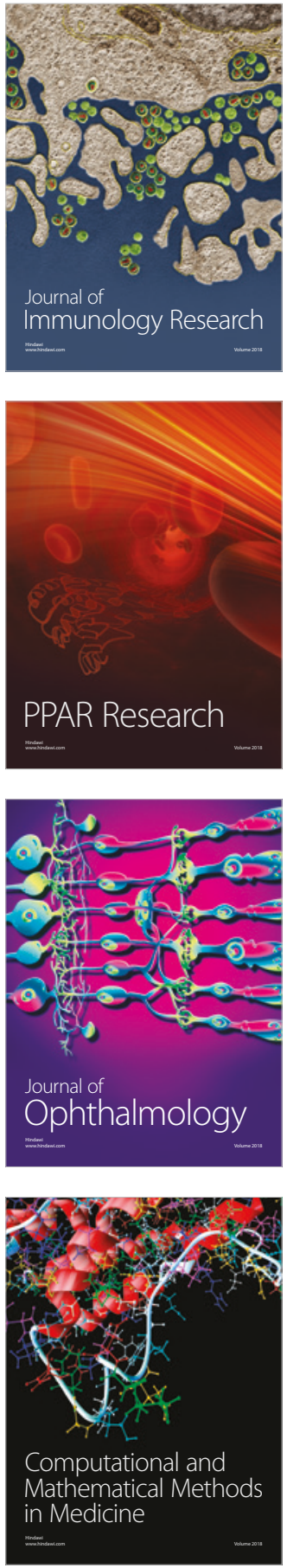

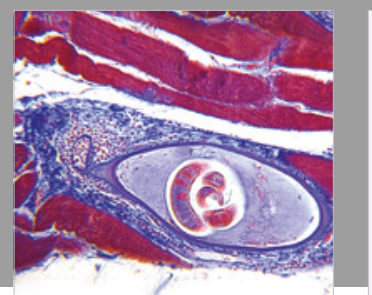

Gastroenterology Research and Practice

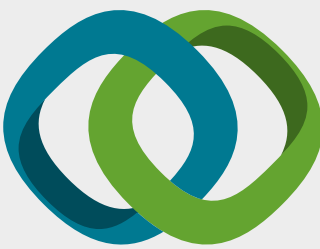

\section{Hindawi}

Submit your manuscripts at

www.hindawi.com
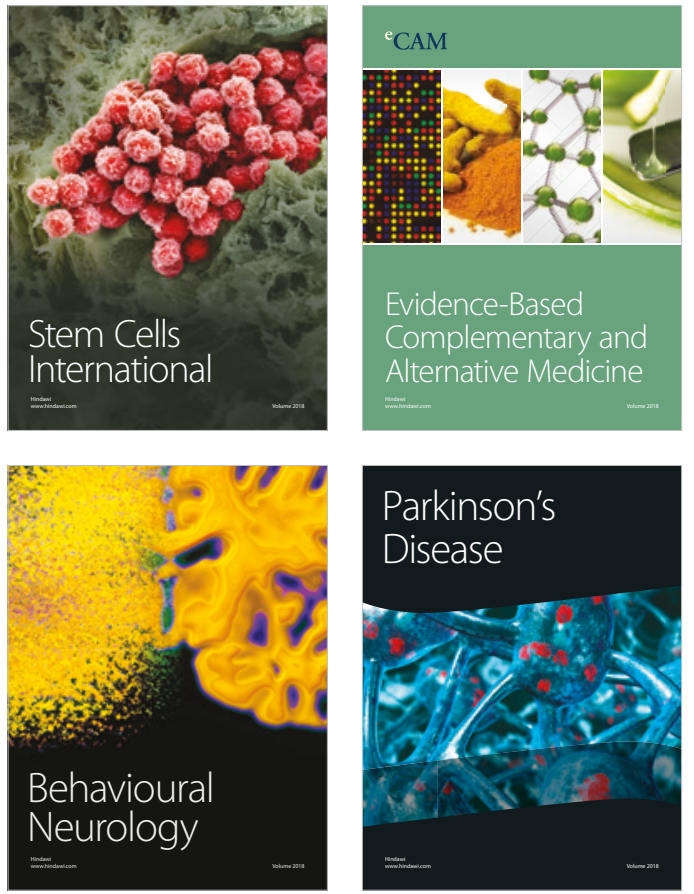

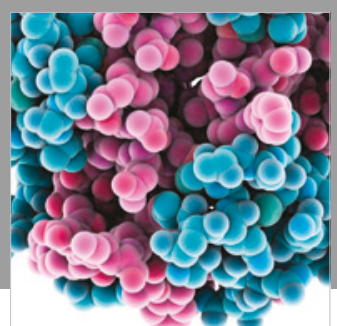

ournal of

Diabetes Research

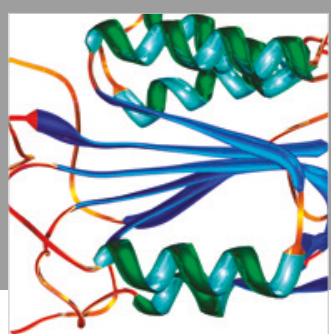

Disease Markers
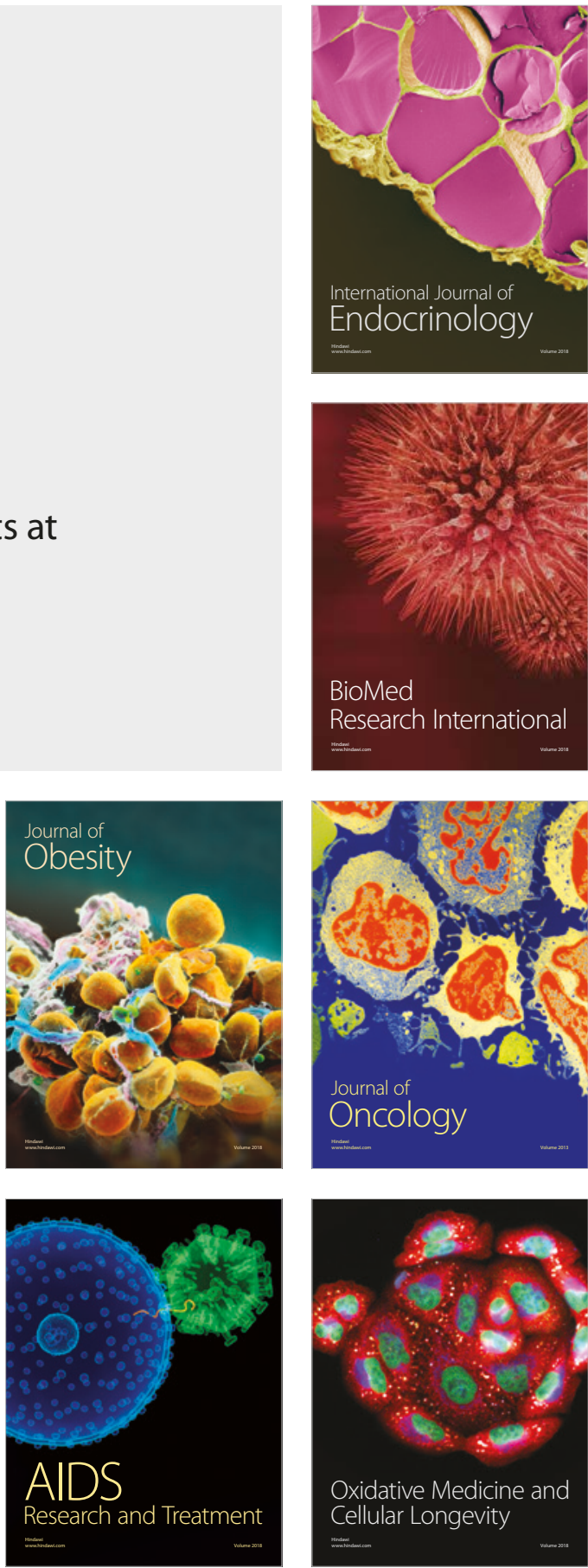